\title{
Retraction Note: The visual object tracking algorithm research based on adaptive combination kernel
}

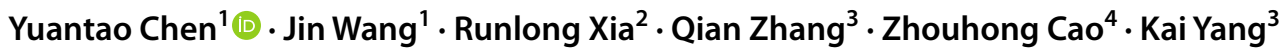

๑) Springer-Verlag GmbH Germany, part of Springer Nature 2021

\section{Retraction to: Journal of Ambient Intelligence and Humanized Computing(2019) 10:4855-4867 https://doi.org/10.1007/s12652-018-01171-4}

The Editor-in-Chief has retracted this article due to significant overlap of the content with (Liu et al. 2018). All authors agree to this retraction.

\section{Reference}

Liu P, Zhang J, Wu D, An Z (2018) Robust video object tracking algorithm based on self-adaptive compound kernel. J Comp Appl 38(12):3372-3379

Publisher's Note Springer Nature remains neutral with regard to jurisdictional claims in published maps and institutional affiliations.

The original article can be found online at https://doi.org/10.1007/ s12652-018-01171-4.

Jin Wang

jinwang@csust.edu.cn

$\triangle$ Runlong Xia

51037560@qq.com

1 Hunan Provincial Key Laboratory of Intelligent Processing of Big Data on Transportation and School of Computer and Communicational Engineering, Changsha University of Science and Technology, Changsha 410114, Hunan, China

2 Hunan Institute of Scientific and Technical Information, Changsha 411105, Hunan, China

3 Technical Quality Department, Hunan ZOOMLION Intelligent Technology Company Limited, Changsha 410005, Hunan, China

4 School of Hydraulic Engineering, Changsha University of Science and Technology, Changsha 410114, Hunan, China 